\title{
Absorption of High Energy Gamma Rays by Interactions with Extragalactic Starlight Photons at High Redshifts
}

\author{
M.H. Salamon* and F.W. Stecker ${ }^{\dagger}$ \\ * Physics Department, University of Utah \\ Salt Lake City, UT 84112 \\ $\dagger$ Laboratory for High Energy Astrophysics \\ NASA/Goddard Space Flight Center \\ Greenbelt, MD 02271
}

\begin{abstract}
We extend earlier calculations of the attenuation suffered by $\gamma$-rays during their propagation from extragalactic sources, obtaining new extinction curves for $\gamma$-rays down to $10 \mathrm{GeV}$ in energy, from sources up to a redshift of $z=3$.
\end{abstract}

The recognition that high energy $\gamma$-rays, propagating over cosmological distances, suffer electron-pair-producing interactions with photons from the extragalactic background radiation fields dates back to the 1960s [1-4]. The reaction $\gamma \gamma \rightarrow e^{+} e^{-}$between a $\gamma$-ray of energy $E$ and a background photon of energy $\epsilon$ can occur when the center-of-mass square energy $s$ is above threshold, $s=2 E \epsilon(1-\cos \theta)>4 m_{e}^{2} c^{4}$, where $\theta$ is the angle between the two photons' direction vectors. A $\gamma$-ray of energy $E_{\mathrm{TeV}} \mathrm{TeV}$ therefore interacts only with background photons above a threshold energy $\epsilon_{\mathrm{thr}} \approx 0.3 \mathrm{eV} / E_{\mathrm{TeV}}$. Since the number density of background photons decreases roughly as a power law in energy, most of the collisions occur near threshold. Thus, when estimating the extinction of $1 \mathrm{TeV}$ gamma rays, it is the density of the infrared background which dominates; at $20 \mathrm{GeV}$, however, only UV photons near the Lyman limit can act as targets.

This mechanism was recently invoked [5] to explain why many EGRET blazars are not seen at $\sim \mathrm{TeV}$ energies by ground-based instruments such as Whipple, in spite of the fact that an extrapolation of the EGRET powerlaw spectra places them above the sensitivity limit of these ground-based detectors. The opacity $\tau$ seen by a $\gamma$-ray in its propagation from source to Earth is roughly $\tau \sim N \sigma_{T} d$, where $N$ is the number of target soft photons

(c) 1995 American Institute of Physics 

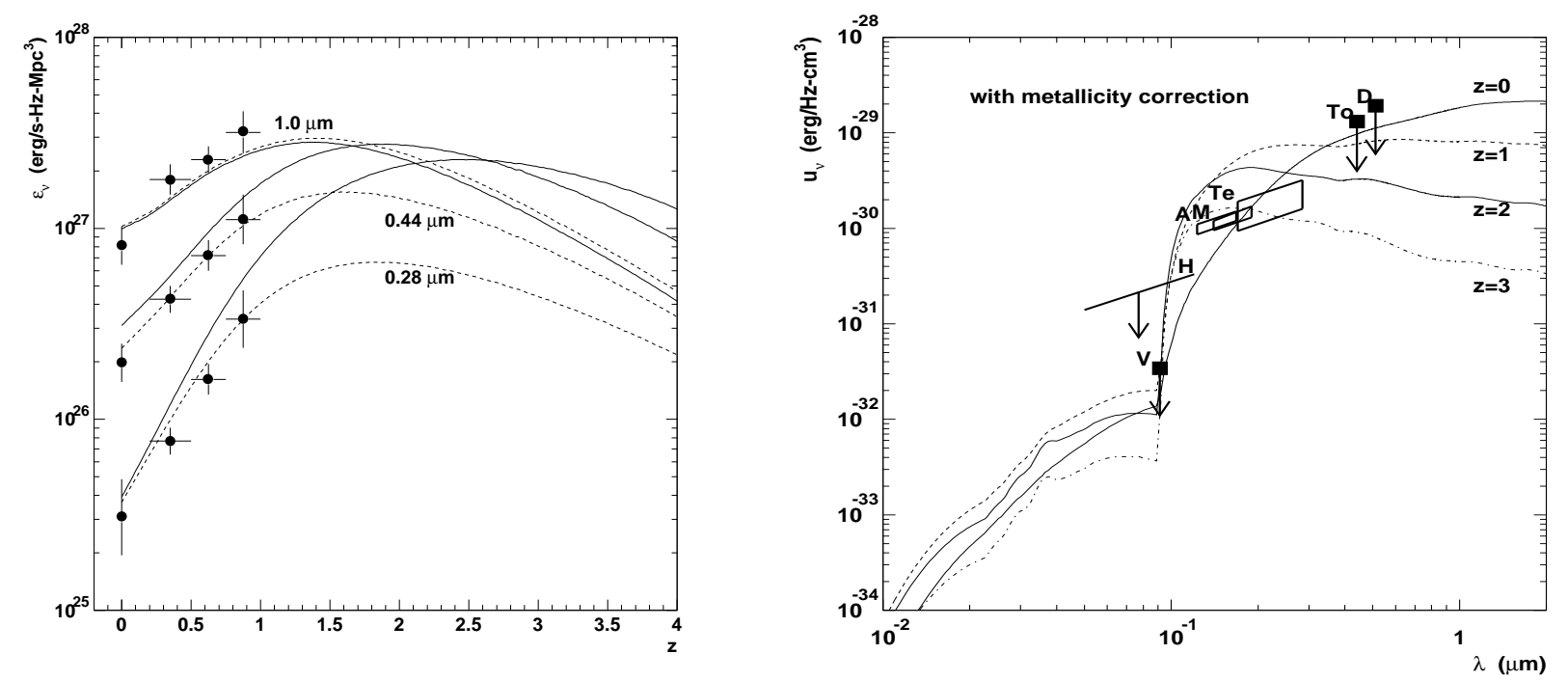

FIGURE 1. Left: The mean co-moving emissivity of stellar populations as a function of redshift for three different wavelengths, with (solid lines) and without (dashed lines) our metallicity correction added. The dashed lines are essentially a reproduction of the results of Ref. [11], and the observational data points are from the Canada-French redshift survey group [13]. Right: The computed co-moving background energy density as a function of wavelength for several redshifts. The data points shown are high galactic latitude detections or limits at redshift $z=0$ : A, Ref. [14]; D, Ref. [15]; H, Ref. [16]; M, Ref. [17]; Te, Ref. [18]; To, Ref. [19]; V, Ref. [20].

above threshold, $\sigma_{T}$ is the Thompson cross section, and $d$ is the distance to the source. For sources with redshift $z>0.1$ (corresponding to most of the EGRET blazar sources), based on estimates of the diffuse IR background [6] the opacity is greater than unity for $\sim \mathrm{TeV} \gamma$-rays, making their ground-based detection unlikely.

With the advent of a new generation of ground-based instruments with anticipated $\gamma$-ray energy thresholds as low as $20 \mathrm{GeV}$, and with the likely future launch of GLAST [7] with sensitivity in the range $\sim 0.01$ to $100 \mathrm{GeV}$, it is important to extend the opacity calculations down to the lowest relevant $\gamma$-ray energies. Although efforts along these lines have been made [8,9], very recent work on the evolution of star formation rates with redshift $[10,11]$ justifies a new and more detailed calculation of $\gamma$-ray opacity.

The role played by the extragalactic starlight background (ESB) in the attenuation of $\gamma$-rays from extragalactic sources is defined in the exact expression for the $\gamma$-ray opacity $\tau$,

$$
\tau\left(E_{0}, z_{e}\right)=c \int_{0}^{z_{e}} d z \frac{d t}{d z} \int_{0}^{2} d x \frac{x}{2} \int_{0}^{\infty} d \nu(1+z)^{3} \frac{u_{\nu}(z)}{h \nu} \sigma_{\gamma \gamma}(s),
$$

where $E_{0}$ is the observed $\gamma$-ray energy, $z_{e}$ is the source redshift, $t(z)$ is the 
cosmic time, $x \equiv(1-\cos \theta), \theta$ is the angle between the photons' direction vectors, $\nu$ is the target photon frequency at redshift $z, u_{\nu}(z)$ is the photon energy density per unit frequency at redshift $z$, and $\sigma_{\gamma \gamma}$ is the Bethe-Heitler cross section for $\gamma \gamma \rightarrow e^{+} e^{-}$.

Apart from the uncertainty in cosmological parameters, the only unknown in the above equation is the ESB energy density $u_{\nu}(z)$. This can be determined if the mean emissivity per unit frequency, $\mathcal{E}_{\nu}(z)$, of starlight from galaxies is known:

$$
u_{\nu}(z)=\int_{z}^{z_{\max }} d z^{\prime} \frac{d t}{d z} \mathcal{E}_{\nu^{\prime}}\left(z^{\prime}\right) e^{-\tau_{\text {cloud }}\left(\nu, z, z^{\prime}\right)}
$$

Here $z_{\max }$ is the redshift for the turn-on of star formation, $\nu^{\prime}=\nu\left(1+z^{\prime}\right) /(1+z)$, and the last factor accounts for the partial absorption of the starlight by intervening Ly $\alpha$ clouds during the ESB's propagation through intergalactic space $[12]$.

The mean emissivity $\mathcal{E}_{\nu}(z)$ is the total stellar energy output per unit volume and frequency, averaged over all galaxies and proto-galaxies, at a given redshift. Consider a population of stars all born at the same instant, with an initial mass function (IMF) $\phi(M) d M \propto M^{-\alpha} d M(\alpha=2.35$ here). The total emission $S_{\nu}(T)$ from this population is the integral of the spectral energy output of each star (a function of its mass $M$, age $T$, and to a smaller extent its metallicity $[21,22])$ weighted by the IMF. As the age $T$ of the population increases, $S_{\nu}(T)$ becomes redder, due to the shorter lifetimes of the bluer stars. The mean emissivity $\mathcal{E}_{\nu}(z)$ is then the convolution of $S_{\nu}(T)$ with the redshift-dependent stellar formation rate, $\dot{\rho}_{s}(z)$ :

$$
\mathcal{E}_{\nu}(z)=\mathcal{T}_{d, g}(\nu) \int_{z}^{z_{\max }} d z^{\prime} \frac{d t}{d z^{\prime}} \dot{\rho}_{s}\left(z^{\prime}\right) S_{\nu}\left[T=t(z)-t\left(z^{\prime}\right)\right] \mathcal{L}\left(\nu, z^{\prime}\right)
$$

where $\mathcal{T}_{d, g}(\nu)$ is the probability that stellar photons of frequency $\nu$ will escape absorption by dust and gas in their parent galaxy, and $\mathcal{L}(\nu, z)$ is a frequencydependent correction to $S_{\nu}$ which accounts for the increase in stellar metallicities with decrease in $z$.

Figure 1 shows our results for $\mathcal{E}_{\nu}(z)\left(\right.$ Eq.3) and $u_{\nu}(z)$ (Eq.2). For $S_{\nu}(T)$ we have used the population synthesis models of Refs. [23,24,22]; for $\mathcal{L}(\nu, z)$ we have constructed an empirical correction function based on the work of Ref. [21]; the star formation rate $\dot{\rho}_{s}(z)$ comes from the beautiful analysis of Refs. $[10,25]$. (See Ref. [26] for more details.)

With Eq.1, the ESB opacity to $\gamma$-rays is calculated, and shown in Fig.2, both with and without the metallicity correction function $\mathcal{L}$ included. Given the uncertainties associated with $\mathcal{L}$ [26], the true opacities likely lie somewhere between the two sets of curves.

Figure 3 shows the effect of the ESB on $\gamma$-ray propagation from several blazars. Note that the spectral cutoffs occur at lower energies for blazars 

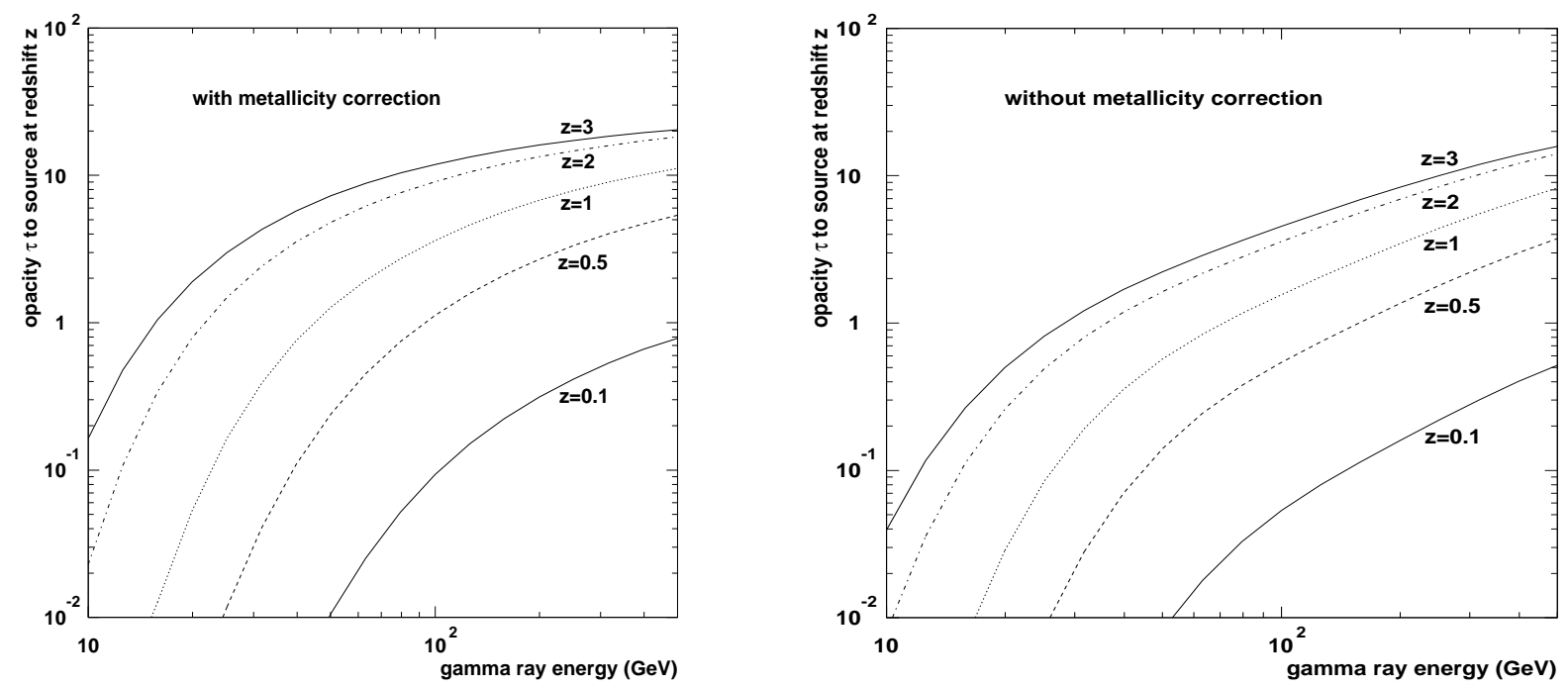

FIGURE 2. The opacity $\tau$ of the ESB to $\gamma$-rays as a function of $\gamma$-ray energy and source redshift $z$. Left: Curves calculated with the metallicity correction included. Right: Curves calculated without metallicity correction. The truth likely lies between these two sets of curves. We note that the opacities obtained here are independent of the value assumed for Hubble's constant (see Ref. [26] for details).

at higher redshifts, a distinctive signature which can discriminate this cutoff mechanism from intrinsic (intrasource) cutoff mechanisms. Also note that there is essentially no attenuation below $10 \mathrm{GeV}$, due to the sharp break in the energy density above the Lyman limit (Fig.1). Figure 3 also shows the beginning of the extinction of that component of the extragalactic $\gamma$ ray background above $20 \mathrm{GeV}$ that is due to unresolved blazars [27]; this is compared with recent EGRET measurements of the extragalactic $\gamma$-ray background [28].

Acknowledgements: We thank M. Fall, M. Malkan, Y.C. Pei, P. Sreekumar, G. Worthey, and N. Wright helpful conversations and advice.

\section{REFERENCES}

1. Nishikov, A.I., Sov. Phys., JETP 14, 393 (1962).

2. Gould, R.J. and Schreder, G.P., Phys. Rev. Lett. 16, 252 (1966).

3. Stecker, F.W., Ap.J. 157, 507 (1969).

4. Fazio, G.G. and Stecker, F.W., Nature 220, 135 (1970).

5. Stecker, F.W., De Jager, O.C., and Salamon, M.H., Ap.J. 390, L49 (1992).

6. Stecker, F.W. and De Jager, O.C., Ap.J. 476, 712 (1997).

7. Bloom, E.D., Sp. Sci. Rev. 75, 109 (1996).

8. Madau, P. and Phinney, E.S., Ap.J. 456, 124 (1996). 

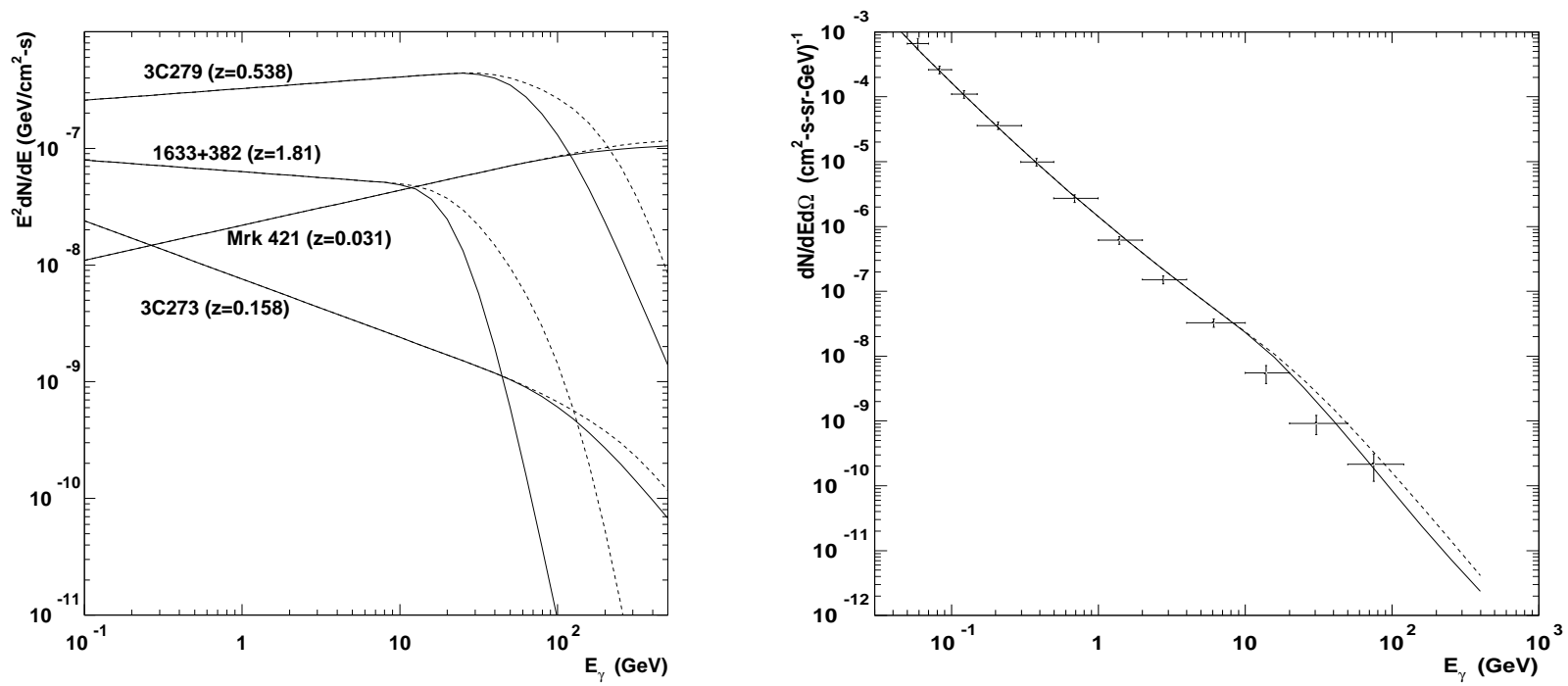

FIGURE 3. Left: The attenuated power-law spectra of four prominent blazars. The solid (dashed) curves are calculated with (without) the metallicity correction factor. Right: Extragalactic $\gamma$-ray background spectrum from unresolved blazars, calculated for the EGRET point source sensitivity of $10^{-7} \mathrm{~cm}^{-2} \mathrm{~s}^{-1}$; solid (dashed) line includes (does not include) metallicity correction, and data points are from EGRET [28].

9. Stecker, F.W. and De Jager, O.C., Sp. Sci. Rev. 75, 413 (1996).

10. Pei, Y.C. and Fall, S.M., Ap.J. 454, 69 (1995).

11. Fall, S.M., Charlot, S., and Pei, Y.C., Ap.J. 464, L43 (1996).

12. Madau, P. and Shull, J.M., Ap.J. 457, 551 (1996).

13. Lilly, S.J., et al., Ap.J. 460, L1 (1996).

14. Anderson, R.C., et al., Ap.J. 234, 415 (1979).

15. Dube, R.R., Wickes, W.C., and Wilkinson, D.T., Ap.J. 232, 333 (1979).

16. Holberg, J.B., Ap.J. 311, 969 (1986).

17. Martin, C., Hurwitz, M., and Bowyer, S., Ap.J. 379, 549 (1991).

18. Tennyson, P.D., et al., Ap.J. 330, 435 (1988).

19. Toller, G.N., Ap.J. 266, L79 (1983).

20. Vogel, S.N., et al., Ap.J. 441, 162 (1995).

21. Worthey, G., Ap.J.Suppl. 95, 107 (1994).

22. Charlot, S., Worthey, G., and Bressan, A., Ap.J. 457, 625 (1996).

23. Bruzual, A.G. and Charlot, S., Ap.J. 405, 538.

24. Charlot, S. and Bruzual, A.G., Ap.J. 367, 126 (1991).

25. Fall, S.M. and Pei, Y.C., Ap.J. 402, 479 (1993). (1993).

26. Salamon, M.H. and Stecker, F.W., submitted to Ap.J. (1997).

27. Stecker, F.W. and Salamon, M.H., Ap.J. 464, 600 (1996).

28. Sreekumar, P., et al., submitted to Ap.J. (1997). 